\title{
ESR mapping of polar-cap patches in the dark cusp
}

\author{
H. C. Carlson, ${ }^{1,2}$ K. Oksavik, ${ }^{3,4}$ J. Moen, ${ }^{2,4}$ A. P. van Eyken, ${ }^{5}$ and P. Guio ${ }^{6}$ \\ Received 18 September 2001; revised 9 February 2002; accepted 15 February 2002; published 21 May 2002.
}

[1] We present the first ever measurement of the full thermal plasma properties, of an ionospheric patch in full darkness in the noon region where patches are believed to form. Further these data present the first experimental evidence for the Lockwood and Carlson class of mechanisms for forming patches by plasma injection. These data were possible only because of a new measurement capability we had to develop. We introduce the capability here because it crosses the high-speed threshold that now allows study of a broader class of mesoscale plasma flow-transients, which are thought to occur over time scales near 2 minutes vice $8-10$ minutes. Cumulatively such transients may significantly drive global convection. We demonstrate both the validity of and need for our new measurement capability, by presenting a transient flow reversal sweeping across a 500 by $1000 \mathrm{~km}$ area, with initial reversal in 4 minutes, and recovery within 6 minutes. INDEX TERMS: 2475 Ionosphere: Polar cap ionosphere; 2463 Ionosphere: Plasma convection; 2724 Magnetospheric Physics: Magnetopause, cusp, and boundary layers; 2431 Ionosphere: Ionosphere/magnetosphere interactions (2736)

\section{Introduction}

[2] Understanding the nature of large-scale transient plasmaflow events near polar regions is essential to understanding the processes that control the character of the polar ionosphere and much of near-earth space. This understanding can also apply to plasma physics more generally. This understanding has further practical application to satellite communications and reception, GPS navigation, and space-based radar imaging. Understanding has until now been hampered by the inability to map rapid changes in polar plasma flow over large areas.

[3] Patches are $100-1000 \mathrm{~km}$ size islands of high density F-region plasma surrounded by significantly lower density plasma. They dominate the character of the polar cap ionosphere half of the time. Plasma densities inside vs. outside ionospheric patches can switch sharply between $10^{10} \mathrm{~m}^{-3}$ and $10^{12} \mathrm{~m}^{-3}$ [Weber et al., 1984], literally night-to-daytime density changes. Reviews of intensive research on patches include Crowley [1996], Dandekar and Bullett [1999], and Basu and Valladares [1999]. The source of the plasma in intense patches is thought to be solar EUV within the sunlit ionosphere. Patches move antisunward across the polar cap, at $\sim 1 \mathrm{~km} / \mathrm{s}$, over a $\sim 5000 \mathrm{~km}$ trajectory across the polar cap, from near noon toward midnight, consistent with a normal two-cell convection pattern [Heelis et al., 1982]. They are then thought to exit from the midnight sector [Pedersen et al., 2000], into subauroral return flow towards noon. They are known to become highly structured over

\footnotetext{
${ }^{1}$ AFOSR, North Randolph Street, Arlington, VA, USA.

${ }^{2}$ Department of Physics, University of Oslo, Norway.

${ }^{3}$ Department of Physics, University of Bergen, Norway.

${ }^{4}$ University Courses on Svalbard, Longyearbyen, Norway.

${ }^{5}$ EISCAT Svalbard Radar, Longyearbyen, Norway.

${ }^{6}$ Institute of Theoretical Astrophysics, University of Oslo, Norway.
}

scale sizes spanning at least 0.1 to tens of $\mathrm{km}$, producing severe scintillation effects [Buchau et al., 1985; Basu and Valladares, 1999] on satellite communication, and GPS navigation signals.

[4] However, we still do not know the dominant mechanism(s) for patch formation [Carlson, 1994; Basu and Valladares, 1999], mostly for want of adequate experimental guidance. Even for the work published in Valladares et al. [1999], which significantly advances understanding of patch formation, the 8 minute cycle resolution in present use does not constrain the results to conclusions as definite as desired.

[5] A related but broader scientific issue relates to global high latitude plasma convection, whose time-averaged behavior is well parameterized and modelled [Heelis et al., 1982; Heppner and Maynard, 1987]. These smooth-flow models have been extremely useful for many purposes, even for modelling some aspects of patch behavior [Anderson et al., 1988; Valladares et al., 1996]. However, there is disagreement on the extent to which plasma and magnetic flux enter the polar cap in disjoint steps (of only minutes duration) vs. smooth flow [Cowley and Lockwood, 1992; Lockwood et al., 1995; Onsager and Elphic, 1996; Smith and Lockwood, 1996], a fundamental question. Measurement of plasma properties over selected large areas, with adequate time resolution, would directly address essential physics and the role of transient flow events in maintaining quasi steady-state transpolar flow, and reconfiguration between different states.

\section{Observing Mode}

[6] Required area mapping and repeat rates, and area coverage, are determined by the present view of reconnection events, convection reconfiguration scales, and plasma signatures of physical processes. Present theory and data set this measurement goal at $\sim 2$ minutes time resolution over areas $>500 \times 500 \mathrm{~km}$. Full plasma densities $(\mathrm{Ne})$, electron $(\mathrm{Te})$ and ion (Ti) temperatures, and velocities (Vi) must all be measured to provide clear process signatures and discriminate between competing theories.

[7] Incoherent scatter radars (ISR) can map large-scale plasma electrodynamics and many key plasma properties [Carlson, 1996] over large areas. Electric fields map directly along magnetic field lines B in the ionosphere above altitudes of $\sim 150 \mathrm{~km}$. Thus low elevation ISR conical scans can project electric fields and plasma velocities to a common reference altitude over large areas. Mapping can be extended below $150 \mathrm{~km}$ in altitude using a model for the neutral atmosphere and ion-neutral collisions. Strong thermal conductivity makes it relatively easy to map electron temperatures along B for topside F-region altitudes. Ion temperatures exceed low neutral gas temperature below $200 \mathrm{~km}$ when experiencing strong joule heating. All three parameters help identify plasma boundaries. Electron density gradients in range can identify some boundaries. Feasibility of applications hinges on signal to noise ratios and statistical error bars.

[8] The new EISCAT Svalbard Radar (ESR) observing mode described here is designed to address this challenge: the entry of ionospheric patches into the polar cap, and large-scale plasma flow reconfigurations. The ESR antenna is scanned in azimuth at a fixed radar elevation angle of $30^{\circ}$, scribing a conical surface. This scan is repeated with a full sweep time of 2 minutes 8 seconds, in successive windshield-wiper like alternate directions of motion. Data was recorded every 3.2 seconds, for analysis at either 3.2 or 
16 second integration periods. The coded pulse length was $50 \mathrm{~km}$, with all transmitter power into the single steerable antenna. The azimuth scan angle was centered on the area to be studied (here the cusp), spanning a nominal $90^{\circ}$ of azimuth.

[9] Design criteria were the following. The ISR must revisit plasma in its field of view (FOV) frequently enough to track positions of recognizable plasma features and discontinuities over time. The ISR scan speed is determined by the horizontal resolution needed, traded against the scan area desired, within constraints of signal strength and sampling rate controlling error bars. The error bars only need to be small relative to parameter differences across boundaries for boundary tracking, but must be much smaller to test for signatures of physical processes. Continuous scanning, vs. a series of fixed positions, gives uniform weight to all plasma within the volume sampled, to minimize distortion (unrepresentative samples) of findings in highly-structured plasma. The coverage area must be great enough to put features in a clear spatial context, to guide theory and models. Instrumental constraints are earth curvature and signal-to-noise ratio. To optimize the latter, we analyzed data with height resolution of a neutral scale height or less (range resolution $50 \mathrm{~km}$ ).

[10] Initial velocities of the ionospheric feet of reconnected flux tubes move at up to $2 \mathrm{~km} / \mathrm{s}$, over distances of many hundreds of $\mathrm{km}$. The radar scan moves through the $\mathrm{F}$ region at about $8 \mathrm{~km} / \mathrm{s}$ at $325 \mathrm{~km}$ altitude. Near-earth satellites move along their orbit at $\sim 7 \mathrm{~km} / \mathrm{s}$. At $325 \mathrm{~km}$ altitude, this scan covers about $1000 \mathrm{~km}$ in width, over a $90^{\circ}$ arc, in the nominal two minutes of scan time we allowed. Signal-to-noise ratios gave F-region ground-range coverage to $600 \mathrm{~km}$ or more, extended to $900 \mathrm{~km}$ if one includes E-plus F-region. The mode gives accurate identification of boundaries between adjoining plasma populations, and plasma properties with sufficient accuracy to discriminate between processes.

\section{Discussion of Data}

[11] The data in Figure 1 show, for every second scan to accent frame-to-frame movement, plasma properties measured along a conical scan, of $30^{\circ}$ elevation angle. The upper half shows plasma density (Ne), velocity (Vi), and ion temperature (Ti), for two times; the lower half shows plasma density and velocity for three times. Positive velocity (red) is flow away from the ESR, negative (blue) is towards. Both scan regions are centered on magnetic east (Geomagnetic north is $32^{\circ}$ west of geographic north).

[12] First consider the data as applied to patch formation. A patch, if formed by the mechanism of a transient magnetic flux tube reconnection event as proposed by Lockwood and Carlson [1992], must exhibit telltale signatures in Vi and Ti. Just after the reconnection event, and until the initial strongest magnetic tension force abates, there would be a velocity spurt, up to $2 \mathrm{~km} / \mathrm{s}$. The force exerted on the flux tube would be externally applied from the solar wind, with the magnetic tension force gripping the magnetic flux tube leading to frictional or joule heating of the ions at the ionospheric feet of the flux tube dragged through the thermosphere. Initially, down-going electrons trapped within the previously closed magnetic flux tube would empty into the thermospheric sink below, and present an extra spurt of electron flux heating of the ambient electrons (Te). A little downstream where the electron heating flux expires, Te relaxes with a time constant of 30 seconds.
Somewhat farther from the region of origin, the velocity spurt and Ti signatures will relax, leaving only the high-density plasma signature commonly seen deeper in the polar cap.

[13] The upper half of Figure 1, shows the first plasma measurements to be made of a patch in the dark polar cusp. The ESR data unambiguously define the feature as a patch, i.e. an island of highdensity plasma entirely surrounded by low-density plasma. For ease of discussion we have degraded the angular resolution of the azimuth scan to a set of ten fan segments of $6^{\circ}$ each. Counting clockwise from the north-most $\mathrm{Ne}$ fan segment, the scan shows a plasma density enhancement in fan-beams $5-7$. Two scans later the same plasma density enhancement feature has moved to fanbeams $3-5$. This corresponds to $100 \mathrm{~km}$ motion of an $\sim 100 \mathrm{~km}$ wide patch. The full time sequence of scans showed uniformly low density initially. Then a high density feature entered the FOV of the ESR, maintained a persistent coherent signature while in the field of view, and finally passed out of view leaving uniform low density plasma again behind it. Thus this stand-alone measurement determined the plasma feature to be an island of high $\left(4 \times 10^{11} \mathrm{~m}^{-3}\right)$ density plasma, completely surrounded by low $\left(<10^{11} \mathrm{~m}^{-3}\right)$ density plasma, i.e. a patch. This too is an experimental first.

[14] Vi shows a flow shear line cutting north-east south-west across several fan-beams, with $\sim 1 \mathrm{~km} / \mathrm{s}$ flow towards the ESR, clockwise of the shear line, and weak away flow on the other side of the shear line. At the earlier time this shear line coincides with the right hand edge of the patch. At the later time, the shear line has moved to the north-west about as much as the patch in the $\mathrm{Ne}$ plots. Note that the flow-jets seen by Valladares et al. [1996, 1999] are aligned east-west (geographic), not north-east south-west. The apparent difference is due only to the orientation of the magnetic $\mathrm{L}$ shells at Sondrestromfjord (Valladares) vs Svalbard (here). In both cases the flow shear parallels the L shells.

[15] Now examine Ti. Both times show an enhanced Ti, in an area elongated along the Vi shear line, and co-located with the plasma enhancement at the altitude and range of the observed plasma patch. Te is uniform in the FOV.

[16] The Lockwood and Carlson mechanism, passes the test of all these signatures, for a patch a little downstream from the point of origin. The IMF was steady southward from 05:45 to 06:13 UT, so southward IMF applies to these data, as needed. While the winter data from Sondrestromfjord [Valladares et al., 1996, 1999] show consistency with flow channels decreasing density by chemically carving preexisting tongues of ionization into segments (patches), to the contrary, the Svalbard data here show flow channels increasing density by transporting patches in from the direction of sunlit plasma. This is the first data to directly support the latter mechanism, and the first data collected with sufficient frame-rate to be able to do so.

[17] In addition to these new physical findings, these results demonstrate the need and utility of such data to meaningfully test various competing patch-generating mechanisms [e.g., Valladares et al., 1994; Crowley, 1996].

[18] Now turn attention to the lower half of Figure 1, showing data at about the same time on the next day, 19 January 2001. Shown is every second $128 \mathrm{~s}$ ( $\sim 2$ minute) azimuth scan, between $15-105^{\circ}$ azimuth (geographic north being $0^{\circ}$, magnetic north $-32^{\circ}$ ) at $30^{\circ}$ elevation. Our focus here is the time scale of reconfiguration of large scale flow-transients, and for brevity we show only $\mathrm{Vi}$ and Ne. The applicable IMF (rather stable

Figure 1. (opposite) Freeze-frame images from "movies" of polar plasma densities (Ne), velocities (Vi), and ion temperatures (Ti) are shown as measured by the EISCAT Svalbard Radar (ESR) near local noon in local darkness. These data are projected to a horizontal plane from the conical surface scanned by the ESR, with azimuth scanned at a $30^{\circ}$ elevation. In these maps, the coordinate arc through the data is $80^{\circ}$ latitude, the radial line through the data is $30^{\circ}$ longitude, and the Svalbard islands coastline is also shown. The upper half of the figure maps the motion of a polar patch in $\mathrm{Ne}, \mathrm{Vi}$, and $\mathrm{Ti}$, for the second and fourth ESR scan after the patch entered the radar view. Velocities away from the ESR are red, towards are blue. In the lower half, images of Ne and Vi are shown for three ESR scans, showing the first, third, and fifth successive scan after onset of a transient polar convection event. The area mapped with this 2-minute time resolution is $\sim 600 \times 1000 \mathrm{~km}$, and for other look angles would reach the coastlines shown for eastern Greenland and northern Norway. 
EISCAT Svalbard Radar: Jan 18, 2001
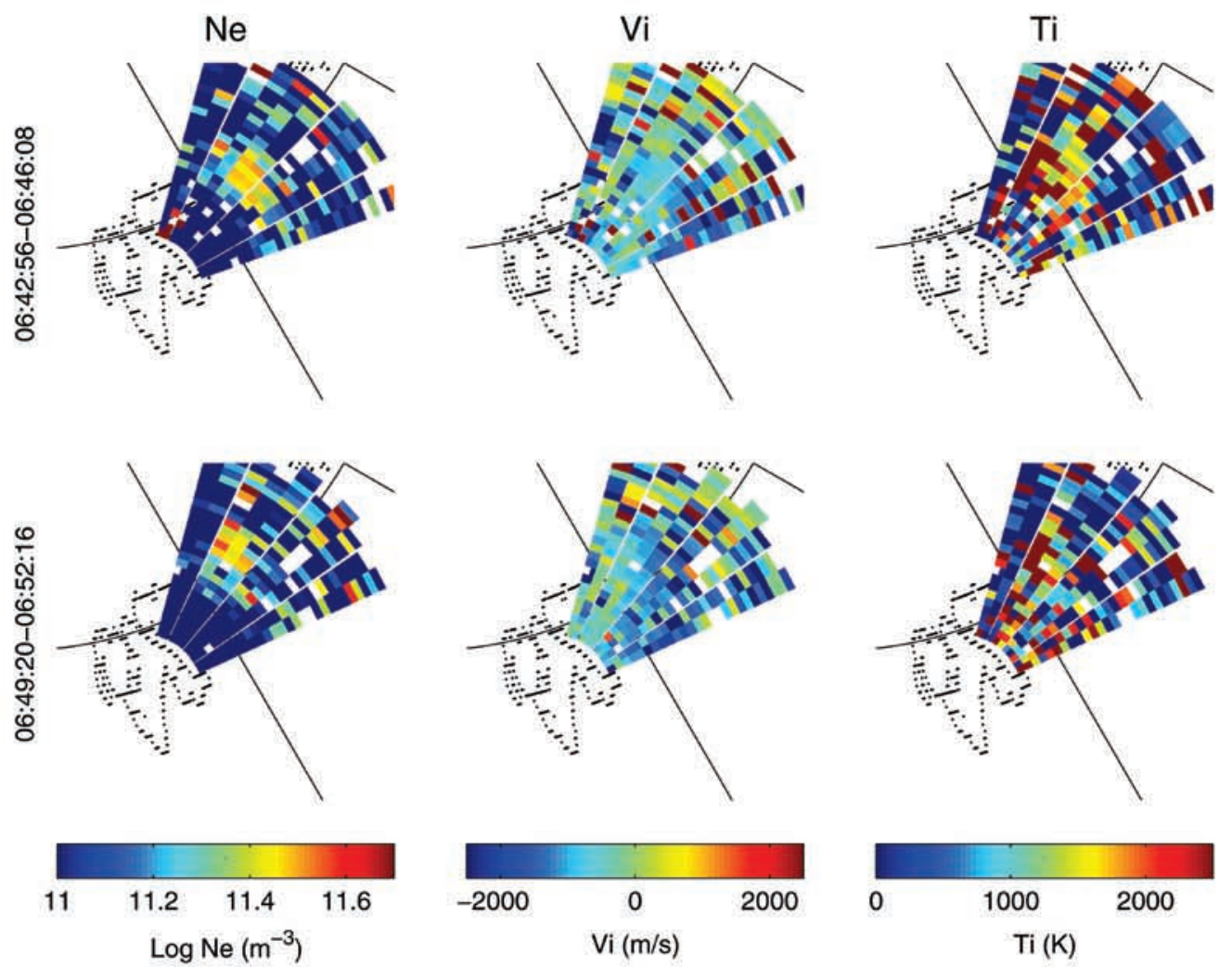

EISCAT Svalbard Radar: Jan 19, 2001
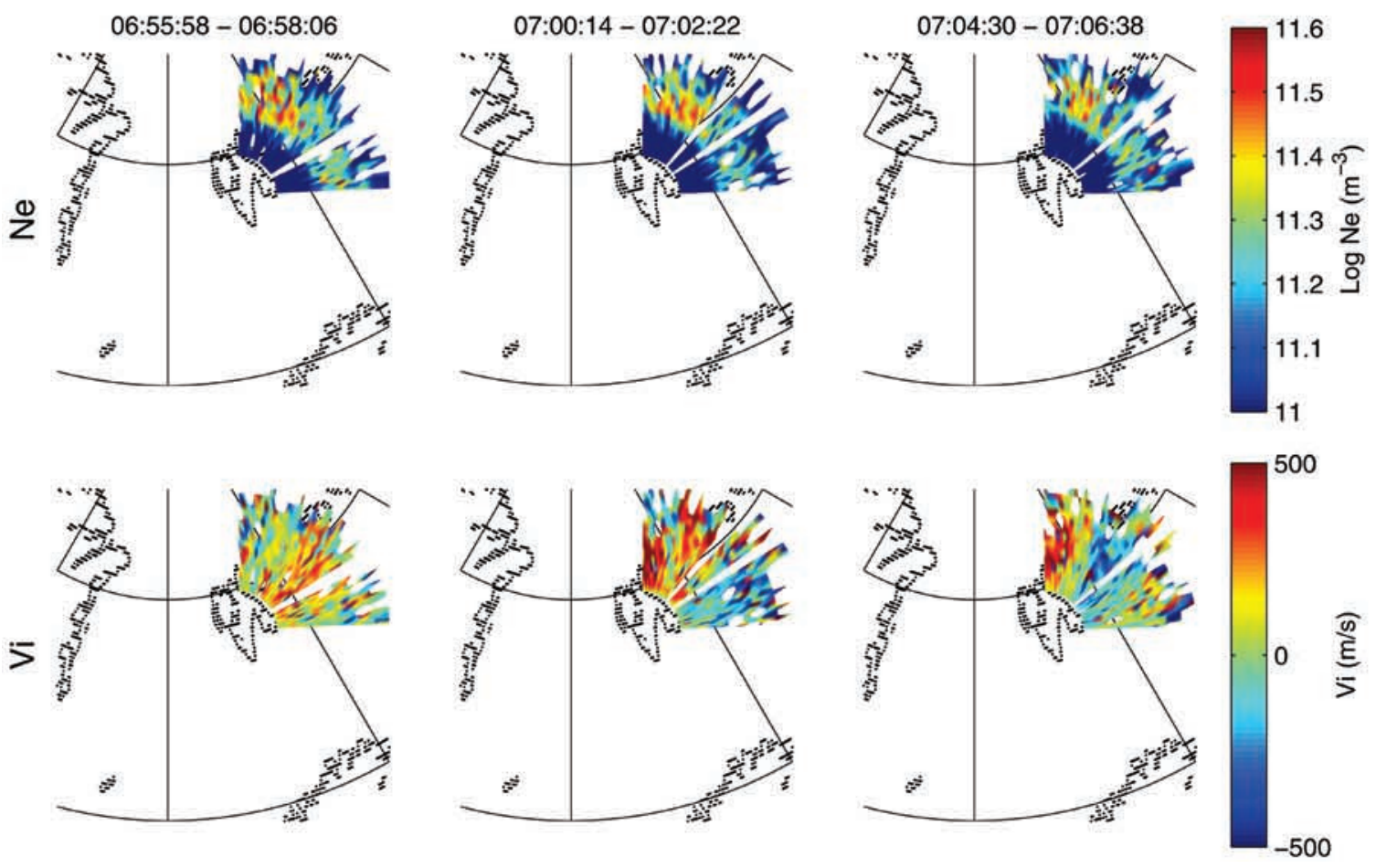
05:37-06:40 UT) was weakly northward, but with the $\mathrm{X}$ component dominant, and clock angle $\sim 45^{\circ}$. At 06:52-06:54 (all times in UT), the ESR FOV was filled with plasma flowing towards (blue) the ESR, a condition to which it returned by 07:06-07:08. Between these times a significant convection reconfiguration and recovery was observed. For brevity we show only image frames of the dynamics of recovery from the initial reconfiguration. At 06:56-06:58 we see reversed flow (red), away from the ESR, across almost the entire FOV. At 07:00-07:02, the southern half of the FOV has returned to towards flow, while the northern half of the FOV is still away flow. By 07:04-07:06, the towards-flow sector has continued to sweep poleward, but with the most poleward sector still away flow. There is $\sim 1 \mathrm{~km} / \mathrm{s}$ difference across the $\sim 200 \mathrm{~km}$ wide shear boundary $(-0.5$ to $+0.5 \mathrm{~km} / \mathrm{s}$ in the ESR rest frame). During 06:52-07:08 UT there were no evident boundaries in Ti or Te (both $<1500 \mathrm{~K}$ ), and only a very modest increase in $\mathrm{Ne}$ within the away (magnetically eastward) flow.

[19] These data illustrate the utility of and need for observing capabilities of the temporal/spatial scales shown here, to study cusp flow transients and convection reconfigurations.

\section{Conclusions}

1. In this paper we have presented the first detection of an ionospheric patch in full darkness in the noon region where patches are believed to form.

2. This is the first stand-alone unambiguous determination of the presence of a patch near its region of origin, and measurement of its thermal plasma properties (density, electron and ion temperatures, and velocity), by any technique. By unambiguous we mean the patch was directly observed to be an island of high plasma-density, fully bounded on all sides by low-density plasma.

3. This is the first measurement in direct support of the class of patch formation mechanisms based on sporadic injection of high density plasma [Lockwood and Carlon, 1992], vs. sporadic chopping of a pre-existing tongue of high density plasma [Valladares et al., 1996, 1999].

4. We introduce a new measurement technique, which finally passes the threshold of 2-3 minute image-frame-rate, specifying thermal plasma properties across mesoscale areas. This time resolution opens the door to testing for ionospheric transient events on time scales order one vs. ten minutes, and their significance to global convection.

5. We illustrate successful passing of this high-speed threshold, and validate its need, by presenting the first 2 minute frame sequence, illustrating transient velocity reversals spanning $>500 \mathrm{~km}$ by $500 \mathrm{~km}$, with reversal onset in 2 minutes and full recovery of initial velocity field in the next 6 minutes.

[20] Acknowledgments. Thanks to staff on EISCAT Svalbard Radar. EISCAT is an International Association supported by Finland (SA), France (CNRS), the Federal Republic of Germany (MPG), Japan (NIPR), Norway (NFR), Sweden (NFR) and the United Kingdom (PPARC). We are happy to acknowledge support from AFOSR 2311AS and the Norwegian Research Council.

\section{References}

Anderson, D. N., J. Buchau, and R. A. Heelis, Origin of density enhancements in the winter polar cap ionosphere, Radio Sci., 23, 513, 1988.

Buchau, J., E. J. Weber, D. N. Anderson, H. C. Carlson, and J. G. Moore, Ionospheric structures in the polar cap: Their origin and relation to $250-$ MHz scintillation, Radio Sci., 20, 325, 1985.

Basu, Sa, and C. Valladares, Global aspects of plasma structures, J. Atm. Solar-Terr. Phys., 61, 127-139, 1999.

Carlson, H. C., The dark polar ionosphere: progress and future challenges, Radio Sci., 29, 157, 1994.

Carlson, H. C., Incoherent scatter radar mapping of polar cap electrodynamics, J. Atm. Solar-Terr. Phys., 58, 37-56, 1996.

Cowley, S. W. H., and M. Lockwood, Excitation and decay of solar-winddriven flows in the magnetosphere-ionosphere system, Ann. Geophys., 10, 103, 1992

Crowley, G., Critical review of ionospheric patches and blobs, Review of Radio Science 1993-1996, Oxford University Press, 619-648, 1996.

Dandekar, B. S., and T. W. Bullett, Morphology of polar-cap patch activity, Radio Sci., 36, 1187-1205, 1999.

Heelis, R. A., J. K. Lowell, and R. W. Spiro, A model of the high-latitude ionospheric convection pattern, J. Geophys. Res., 87, 6339, 1982.

Heppner, J. P., and N. C. Maynard, Empirical high-latitude electric field models, J. Geophys. Res., 92, 4467, 1987.

Lockwood, M., and H. C. Carlson, Production of polar cap electron density patches by transient magnetopause reconnection, Geophys. Res. Lett., 19, 1731-1734, 1992.

Lockwood, M., S. W. H. Cowley, M. F. Smith, R. P. Rijnbeek, and R. C. Elphic, The contribution of flux transfer events to convection, J. Geophys. Res., 22, 1185-1188, 1995.

Onsager, T. G., and R. C. Elphic, Is magnetic reconnection intrinsically transient or steady state? The earth's magnetopause as a laboratory, EOS Trans, $A G U, 77,241-245,1996$.

Pedersen, T. R., B. G. Fejer, R. A. Doe, and E. J. Weber, An incoherent scatter radar technique for determining two-dimensional horizontal ionization structure in polar-cap F-region patches, J. Geophys. Res., 105, $10,637-10,655,2000$.

Smith, M. F., and M. Lockwood, Earth's magnetospheric cusps, Rev. of Geophys., 34, 233-260, 1996.

Valladares, C. E., Su Basu, J. Buchau, and E. Friis-Christiansen, Experimental evidence for the formation and entry of patches into the polar cap, Radio Sci., 29, 167-194, 1994.

Valladares, C. E., D. T. Decker, R. Sheehan, and D. N. Anderson, Modeling the formation of polar cap patches using large plasma flows, Radio Sci., 31, 573-593, 1996.

Valladares, C. E., D. Alcayde, J. V. Rodreguez, J. M. Ruohoniemi, and A. P. Van Eyken, Observation of plasma density structures in association with the passage of traveling vorticies and the occurrence of large plasma jets, Ann. Geophys., 14, 1020-1039, 1999.

Weber, E. J., J. J. Buchau, J. G. Moore, J. R. Sharber, R. C. Livingston, J. D. Winningham, and B. W. Reinisch, F-layer ionization patches in the polar cap, J. Geophys. Rcs., 89, 1683, 1984.

H. C. Carlson, AFOSR, 801 N. Randolph St., Arlington, VA 22201, USA. (herb.carlson@afosr.af.mil)

A. P. van Eyken, EISCAT Svalbard Radar, N-9170 Longyearbyen, Norway. (Tony.van.Eyken@eiscat.com)

P. Guio, Institute of Theoretical Astrophysics, University of Oslo, P.O.Box 1029 Blindern, N-0315 Oslo, Norway. (patricg@fys.uio.no)

J. Moen, Department of Physics, University of Oslo, P.O. Box 1048 Blindern, N-0316 Oslo, Norway. (jmoen@fys.uio.no)

K. Oksavik, Department of Physics, University of Bergen, Allégaten 55, N-5007 Bergen, Norway. (kjellmar.oksavik@fi.uib.no) 